\title{
Obstetric outcomes really worse with the induction of labor
}

\begin{abstract}
Objective: to describe the obstetric outcomes in patients with an active induction of labor (IOL).

Methods: 1037 deliveries were included. In case of IOL, women with unfavorable cervix (Bishop $\leq 6)$ start with 2 tablets of $25 \mathrm{mcg}$ vaginal misoprostol followed by a new tablet every 4 hours up to a maximum of 4 doses. In case of any contraindication for misoprostol a 10mg tablet of dinoprostone was inserted into the vagina. In any case, a cardiotocographic record during 30 minutes was performed previous to each dose. When Bishop was $>6$, artificial rupture of membranes and oxytocin stimulation was performed.
\end{abstract}

Results: Of 351 spontaneous deliveries, 57 (16.24\%) were urgent cesarean section, $211(60.11 \%)$ were eutocic, $58(7.12 \%)$ ended by forceps and $58(16.52 \%)$ by vacuum. Among the IOL (556), 127 (22.84\%) completed the delivery by urgent cesarean section, $291(52.33 \%)$ were eutocic, $55(9.89 \%)$ ended by forceps and $81(14.56 \%)$ by vacuum
Volume 12 Issue 2 - 202I

\author{
Jorge Duro Gómez,' Justo Martínez León,2 \\ Ana Belén Rodríguez Marín,' Rosa María \\ Llamas Fuentes \\ 'Department of Gynecology and obstetrics, San Juan De Dios \\ Hospital, Spain \\ ${ }^{2}$ Midwife, San Juan De Dios Hospital, Spain
}

Correspondence: Jorge Duro Gómez, Department of Gynecology and obstetrics, San Juan De Dios Hospital, Córdoba, C/Arabista Joaquina Eguaras $N^{\circ} 2$ Esc $4 I^{\circ} \mathrm{B}$, Spain,

Tel+34685810803, Email jorgedurogomez@gmail.com

Received: March 09, 202I | Published: April |4, 202 I

Conclusion: the active attitude during the IOL favors results similar to those when the delivery is spontaneous.

Keywords: obstetric outcomes, induction of labor, maternal, fetal

\section{Introduction}

Induction of labor (IOL) represents an opportunity to intervene in an ongoing pregnancy in order to start the delivery timing for either maternal or fetal benefit. ${ }^{1}$ However, currently there may be another reason for the IOL. The elective IOL chosen by the women or IOL for geographical reason are some of the examples.

In 2018, Philipa concluded in a systematic review than IOL at or beyond term compared with expectant management is associated with fewer perinatal deaths and fewer caesarean sections. Since then, many articles have been published on the advisability or not of promoting induction of labor in term fetuses. ${ }^{2}$ In addition, many are the patients who claim this elective IOL.

Consequently, the incidence of IOL is increasing in recent decades. ${ }^{3}$ Currently, average rates of induction of labor (IOL) based on international studies are approximately $20-25 \%$ of all pregnancies. ${ }^{4}$

The aim of this study was to describe the obstetric outcomes in patients with an active IOL.

\section{Methods}

All deliveries at San Juan de Dios Hospital in Córdoba during 2020 were included. The type of initiation and ending of delivery was analyzed. Scheduled cesarean sections were excluded as well as those patients who had a contraindication for vaginal delivery.

In case of IOL, women with unfavorable cervix (Bishop $\leq 6)$ start with 2 tablets of $25 \mathrm{mcg}$ vaginal misoprostol followed by a new tablet every 4 hours up to a maximum of 4 doses. In case of any contraindication for misoprostol a $10 \mathrm{mg}$ tablet of dinoprostone was inserted into the vagina. In any case, a cardiotocographic record during 30 minutes was performed previous to each dose. When Bishop was $>6$, artificial rupture of membranes and oxytocin stimulation was performed.
For the statistical calculation, the freely available program G-Stat 2.0 was used.

\section{Results}

1037 deliveries were included. 130 were elective caesarean sections $(12.53 \%), 351$ (33.84\%) start labor spontaneously and 556 were IOL $(53.61 \%)$

Of 351 spontaneous deliveries, 57 (16.24\%) were urgent cesarean section, 211 (60.11\%) were eutocic, 58 (7.12\%) ended by forceps and $58(16.52 \%)$ by vacuum (Figure 1$)$.

\section{SPONTANEOUS LABOUR}

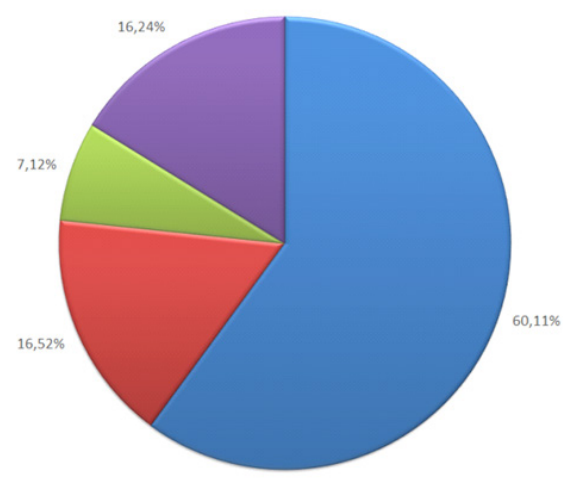

EUTOCIC —CESAREAN SECTION FORCEPS VACUUM

Figure I Spontaneous labour.

Among the IOL (556), 127 (22.84\%) completed the delivery by urgent cesarean section, 291(52.33\%) were eutocic, $55(9.89 \%)$ ended by forceps and $81(14.56 \%)$ by vacuum (Figure 2). A serious complication occurred in a vacuum delivery that ended in puerperal 
hysterectomy due to puerperal metrorrhagia refractory to conservative treatments.

IOL

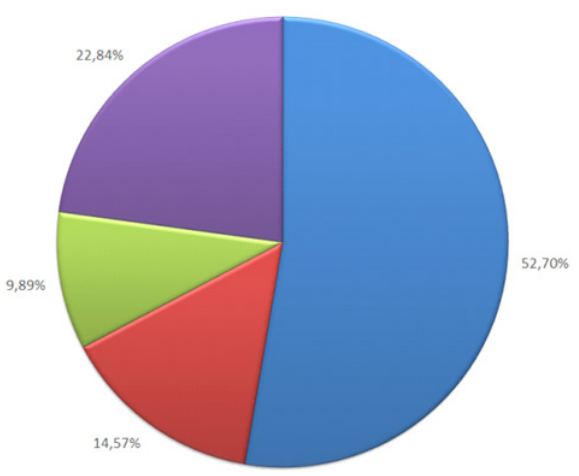

EUTOCIC —CESAREAN SECTION FORCEPS — VACUUM

Figure $2 \mathrm{IOL}$.

\section{Discussion}

According to the work published by Gumelzoglu in $2012^{5}$ a policy of IOL compared with expectant management is associated with fewer perinatal deaths and fewer caesarean sections. Some infant morbidities such as meconium aspiration syndrome were also reduced with a policy of post-term labour induction although no significant differences in the rate of NICU admission were seen. However, from an obstetric point of view, we should not only be interested in neonatal outcomes but also in delivery outcomes. For this reason, as Gumerzoglu concludes, we think women should be appropriately counselled in order to make an informed choice between scheduled induction for a post-term pregnancy or monitoring without induction. In this sense, having the own results relative to each hospital is useful and truthful information.

More important risk factors to have into a count for dissatisfaction of our women could be emergency cesarean section, instrumental vaginal delivery postpartum hemorrhage and Apgar score $<7$ at five minutes, epidural anesthesia, increased medical induction of childbirth are associated with dissatisfaction in women.

According to Middleton results there is a clear reduction in perinatal death with a policy of IOL at or beyond 37 weeks compared with expectant management, though absolute rates are small $(0.4$ versus 3 deaths per 1000). There were also lower caesarean rates without increasing rates of operative vaginal births and there were fewer NICU admissions with a policy of induction. In our study, we can see that operative vaginal birth are similar are similar in both groups (about $24 \%)^{6}$

For the success of the IOL it should involve a coordinated team approach involving the obstetrical provider, specialty trained obstetric nurse, anesthesia staff, and a neonatology team. Only with personalized attention and the right staff can we maintain an active attitude during the IOL. For this reason it is possible that our practice cannot be carried out in all hospitals. Success and failure of IOL Success or failure can be non-opposite concepts: in general, the success of the induction of labor must be represented by the achievement of a vaginal delivery, even if it is operative, without a defined time limit.

We wonder if we should offer the mother the opportunity to opt for an IOL from week 37 or to maintain an expectant attitude. In our opinion, it is essential to involve the patient in the decision after being informed. It is very important for optimal results that our patients to have a positive and conciliatory attitude. In this sense, maternal educational during the pregnancy is a good option. For this reason, during pregnancy women can access 5 training sessions where.

We can conclude that the active attitude during the IOL favors results similar to those when the delivery is spontaneous. Current studies such as this one show that in certain circumstances IOL is not always accompanied by worse perinatal results.

\section{Acknowledgments}

None.

\section{Funding}

None.

\section{Conflicts of interest}

The author declares that there is no conflict of interest regarding this study.

\section{References}

1. Gill P, Lende MN, Van Hook JW. Induction of Labor. Treasure Island (FL): StatPearls Publishing; 2020 .

2. Middleton $P$, Shepherd E, Crowther CA. Induction of labour for improving birth outcomes for women at or beyond term. Cochrane Database Syst Rev. 2018;5(5):CD004945.

3. Caughey AB, Sundaram V, Kaimal AJ, et al. Maternal and neonatal outcomes of elective induction of labor. Evid Rep Technol Assess (Full Rep). 2009;176:1-257.

4. Brun R, Spoerri E, Schäffer L, et al. Induction of labor and postpartum blood loss. BMC Pregnancy Childbirth. 2019;19(1):265.

5. Gülmezoglu AM, Crowther CA, Middleton P, et al. Induction of labour for improving birth outcomes for women at or beyond term. Cochrane Database Syst Rev. 2012;6(6):CD004945.

6. Middleton P, Shepherd E, Morris J, et al. Induction of labour at or beyond 37 weeks' gestation. Cochrane Database Syst Rev. 2020;7(7):CD004945. 